\title{
Peripheral Blood Flow Velocity and Peripheral Pulse Wave Velocity Measured Using a Clip-type Pulsimeter Equipped with a Permanent Magnet and a Hall Device
}

\author{
Keun-Ho Kim and Sang-Suk Lee* \\ Department of Oriental Biomedical Engineering, Sangji University, Wonju 220-702, Korea
}

(Received 10 February 2015, Received in final form 16 March 2015, Accepted 17 March 2015)

\begin{abstract}
We measured radial arterial pulse signals using a prototype of a clip-type pulsimeter equipped with a permanent magnet and a Hall device, which produced signals through a voltage-detecting circuit. The systolic peak time and the reflective peak time for a temporally pulsed signal were analyzed for an arbitrary pulse wave at one position of a small permanent magnet. The measured value of the peripheral pulse wave velocity was about $1.25-1.52 \mathrm{~m} / \mathrm{s}$, demonstrating the accuracy of this new method. To measure the peripheral blood flow velocity, we simultaneously connected the radial artery pulsimeter to a photoplethysmography meter. The average value of the peripheral blood flow velocity was about $0.27-0.50 \mathrm{~m} / \mathrm{s}$.
\end{abstract}

Keywords : radial artery pulse wave, clip-type pulsimeter, photoplethysmography, systolic peak time, reflective peak time, peripheral blood flow velocity, peripheral pulse wave velocity

\section{Introduction}

Several currently available devices that sense living body signals have a common problem; the output signal values are changed by noise, depending on the position of the measuring sensor. In general, blood flow velocity (BFV) monitored by measuring the signal of a pressurized sensor module in a patient's wrist or forearm does not provide customers with useful information regarding disease or health of the cardiovascular system [1]. Also, the BFV is difficult to measure by noninvasive methods under constant pressure without additional pressurization. Medical devices that provide measurements with low accuracy or reproducibility cannot be used to assist diagnosis or treatment. Therefore, a monitoring medical device that can simply measure $\mathrm{BFV}$, pulse rate, heart rate, blood pressure, and pulse wave velocity (PWV) without pressurization is needed [2].

Hardening arteries and heart attacks are caused by cardiovascular disease, which may be due to changes in our dietary patterns that emphasize meat consumption. Research and development of new medical devices are needed to prevent cardiovascular disease $[3,4]$. Electro-

(C)The Korean Magnetics Society. All rights reserved.

*Corresponding author: Tel: +82-33-730-0415

Fax: +82-33-738-7610, e-mail: sslee@sangji.ac.kr cardiography as a clinical device cannot detect ischemic heart disease at an early stage and cannot forecast the function of BFV for the symptoms of cardiac insufficiency [3]. Also, advanced imaging and a blood vessel examination are needed to show the state of heart's veins and arteries to make a diagnosis of cardiovascular disease. To understand the factors contributing to cardiovascular disease and heart attacks, several investigations of blood flowing special quality and blood state of coronary arteries support not only confirming the state of coronary arteries through imaging, but also through electrocardiogram (ECG) examinations [4].

Arterial pulse waves show several physical cardiac characteristics, including the change of pressure and wave motion along the artery. Pulsimeters record the fluctuation of blood vessels through the cardiac cycle. In the left ventricle during the cardiac contraction period, the cardiac output is distributed to the periphery and the blood volume within the arteries is increased. The inhalation of partial blood from a peripheral organization to heart in the diastolic period exists. The tiny changes in blood volume throughout the cardiac cycle in tissues can be recorded by using biographical, mechanical, optical, and other methods [5].

PWV reflects artery stiffness, which can be useful in determining the prognosis of patients with cardiovascular diseases, and serves as a marker of the severity of athero- 
sclerotic vascular damage. Recently, the brachial-ankle PWV (baPWV), a parameter that is easier to obtain than the conventional carotid-femoral PWV, has proved useful the prognosis of patients with cardiovascular diseases [6]. The baPWV can be obtained simply by wrapping the four extremities with blood pressure cuffs and it can be used to screen a large number of subjects easily [7]. In a subject with high blood pressure, it is difficult to estimate whether PWV reflects solely the level of blood pressure or the risk for cardiovascular diseases, including the severity of atherosclerotic vascular damage, in addition to blood pressure. Therefore, a nomogram of the relation of baPWV related to blood pressure may be useful to more precisely estimate the absolute risk for atherosclerotic cardiovascular disease as assessed based on the baPWV in a subject with high blood pressure $[6,7]$.

Pressure waves happen due to the contraction of the heart's main artery and are delivered to the radial artery through a main aortic artery. The PWV is defined as the distance traveled by the wave from heart to radial artery divided by the time it took to travel that distance [8]. The PWV depends on the degree of elasticity of the blood vessel. It increases as the degree of blood vessel elasticity and systolic blood decrease and as blood pressure increases during the contraction period. Diseases related to the blood vessel, such as hardening of the arteries, high blood pressure, glycosuria, hyperlipemia, and new enteropathy, can be detected at early stages by using the principle of PWV $[9,10]$.

According to the basic waveform and electric generation of the pulse wave, the starting point of a pulse wave is where blood of the left ventricle is rescued when an aortic valve is opened. The peak point of a pulse wave is the fast velocity from the left ventricle to the main artery since maximum capacity of the blood vessel is reached when a contraction period peak point is pressured. Also, the peak point of the reflected wave refers to the rescued amount of blood for the contraction period that influenced the reflection of the pulse wave. The notch peak point refers to a low pressure after having rescued blood when an aortic valve is closed. The peak point of a dicrotic wave next to a reflected wave peak indicates that pressure in the artery is increased by the blockade of aortic valve [8].

In this research, the interrelation of each signal and the special quality of the data of pulse wave measured by a clip-type pulsimeter equipped with a permanent magnet and a Hall device were studied utilizing patient monitoring equipment that displayed both signals of the radial artery pulse (RAP) and the photoplethysmography (PPG) wave $[1,2]$. In particular, the peripheral blood flow velocity (PBFV) and the peripheral pulse wave velocity (PPWV) simultaneously measured using the clip-type pulsimeter and the PPG were investigated.

\section{Experimental Method}

The variation of vessel thickness and the depth of the radial artery from the skin's surface result from the periodic motion of arterial vessel. The periodicity of the pulse vessel has a special quality and relationship to the ECG wave and the BFV changes periodically $[1,2]$. The prototype products used in this research consisted of a permanent magnet, Hall sensor, measurement part, light emitting diode (LED), display panel, USB port, switch etc., as shown in Fig. 1(a). The dimensions of the permanent magnet were a diameter of $2 \mathrm{~mm}$, height of 1 $\mathrm{mm}$, and surface magnetic field of about 300 Oe. A permanent magnet was set in the center position of latex rubber and fixed with epoxy. The latex rubber having a good that hold up on skin contact part used to press radial artery part on the whole evenly. The permanent magnet bound by epoxy in the center of the latex rubber was positioned on the "Chwan" point of the radial artery and the device encloses the wrist with contact across the skin surface, as shown in Fig. 1(b). A cylindrical permanent magnet in contact with the skin of the radial artery moves easily, according to the shock of the pulse. To measure
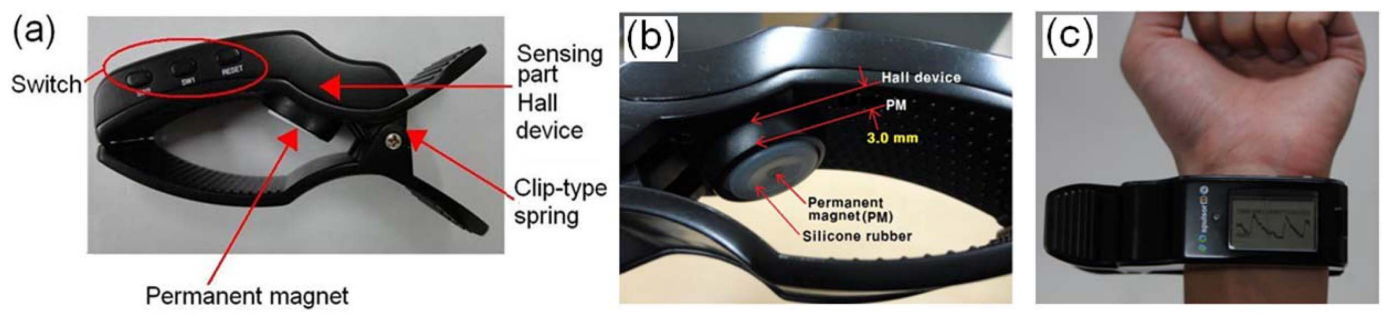

Fig. 1. (Color online) (a) Major parts of the device. (b) The details for the pulse measuring part of clip-type pulsimeter with a permanent magnet (PM), a Hall device, and silicon rubber. The distance between the PM and Hall device is $3.0 \mathrm{~mm}$. (c) The clip-type pulsimeter equipped with a permanent magnet and a Hall device that can be used to measure radial artery pulse. 
(a)
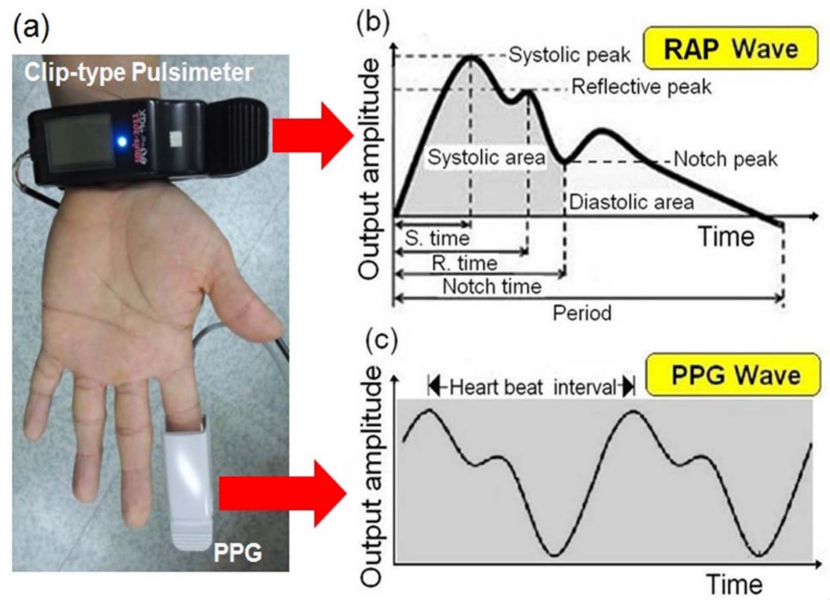

Fig. 2. (Color online) (a) A real feature of two simultaneous measuring devices consisted of one clip-type pulsimeter in the wrist and one photoplethysmography (PPG) device in the index finger. (b) The major parameters, such as the period of pulse wave, the systolic time, the reflective time, and the notch time. (c) The typical waveforms of PPG.

pulse wave in this experiment, the displacement between the Hall device and permanent magnet was $2.5 \mathrm{~mm}$. One permanent magnet in measurement part was moved $1 \mathrm{~mm}$ up and down by periodic motion of radial artery vessel $[10,12]$.

The actual device that was put on the wrist and measured radial artery waveforms is shown in Fig. 1(c). The electrical output signal of the pulse wave acquires self-examination measurements by a clip-type pulsimeter equipped with a Hall device that establishes a base point automatically by the variation of the magnetic field intensity, depending on the fluctuation of the position of the permanent magnet, and the signal passes through a filtering process to eliminate noise. The pulse waveform analysis of 1000 points/s can be transmitted to a PC. The repeated pulse waveforms having 5 sections after selected one section were calculated by a user algorithm program through the clip-type pulsimeter [13, 14].

The PBFV, as shown in Fig. 2, was measured by a cliptype pulsimeter that detected the pulse rate of the radial artery. The absorption of oxyhemoglobin $\left(\mathrm{HbO}_{2}\right)$ in the index finger was measured by a specific photoelectric method, PPG $[7,8]$. The light intensity changes according to the heart beat cycle, penetrating the skin attached to the photodetector. The signal of PPG decreases due to the attenuation of light in tissue as blood flow increases during the contraction period and the signal decreases during the relaxation period $[6,7]$.

Fig. 2(a) shows a device for the measurements of both PBFV and PPWV that uses one molding liquid crystal display (LCD) monitor connected to both the clip-type pulsimeter and the PPG meter. The value of PBFV is defined as the distance from wrist to index finger divided by time difference between the two highest peaks of the RAP and the PPG wave, as shown in Fig. 2(b) and Fig. 2(c). The value of PPWV, on the other hand, is defined as two times distance from wrist to finger divided by the time difference between a systolic peak and a reflected peak of the RAP, as shown in Fig. 2(b).

\section{Results and Discussion}

The status of blood can be known from a blood test, but the special qualities of the coronary artery need to be inspected by the thickness of the vessel, the compliance, and degree of elasticity. The most important thing is to judge the quality of the coronary artery correctly. The coronary artery has several special qualities, such as twitching, convulsion, and extension, unlike other blood vessels. Each special quality of vein and artery has a complicated relationship with the vessel's internal pressure. Therefore, the problems associated with arteriosclerosis that need to be solved are related to the compliance of the blood vessel, the amount of blood flow, the BFV, and the blood flow resistance in the coronary artery [8, 9]. Fig. 3 and Fig. 4 depict the principle and method to measure PBFV and PPWV used to elucidate the special qualities of the coronary artery.

Fig. 3 shows the instrument for PBFV that uses an RAP waveform and PPG pulse waveform. RAP and PPG waves obtained from the simultaneous measurements by the clip-type pulsimeter and the PPG meter mounted on

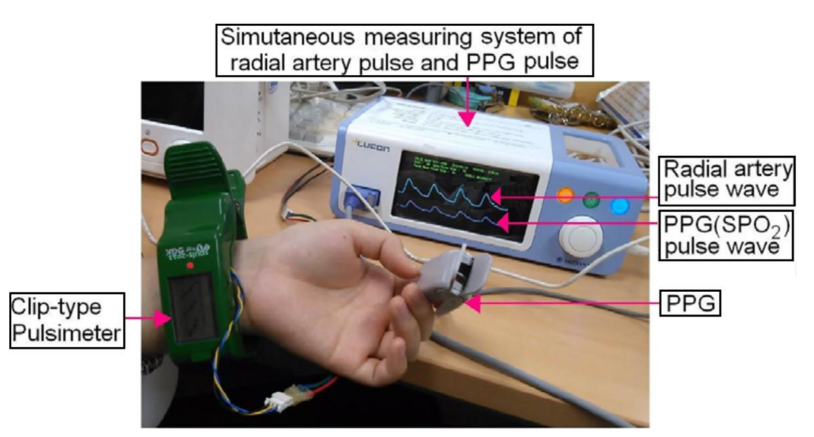

Fig. 3. (Color online) A photograph of the instrument used to measure peripheral blood flow velocity from a radial artery pulse waveform and photoplethysmography (PPG) pulse waveform. Radial artery pulse and PPG waves obtained from the simultaneous measurements by the clip-type pulsimeter and the PPG meter mounted on the wrist and subject's left fingertip, respectively. The PBFV is defined as $L_{H} / \Delta \tau, L_{H}$ is the hand length from wrist artery to index finger and $\Delta \tau$ is the time interval between two peaks of pulse waveforms. 
the wrist and index fingertip of subject's left hand, respectively. The PBFV is defined as $L_{H} / \Delta \tau, L_{H}$ is the hand length from wrist artery to index finger and $\Delta \tau$ is a time interval between peaks of two pulse waveforms. Two waveform differences that compare capacity pulse wave elder brother gotten by $\mathrm{SPO}_{2}$ (oxygen saturation) or PPG that display clip-type pulsimeter and oxygen saturation of the radial artery getting by a patient's artery status that Hall device is installed at the same time as shown in Fig. 4.

The definitions for several major parameters of the pulse wave needed for the calculations of PPWV and PBFV are shown in Fig. 2(a), including systolic peak time (S.time), reflective peak time (R.time), notch amplitude time (N.time), and period time (P.time). As shown in Fig. 4, two pulse waveforms to detect the pulse wave of the user are electronically connected to the display monitor. The PBFV can be calculated $L_{H}$ of a hand length from artery wrist to index finger divided into $\Delta \tau$ that is two waveforms highest peak times and can also be used to estimate blood pressure by analyzing these results $[2,14]$.

The measured values of systolic reflection time, arterial PPG time, hand length, PBFV, and PPWV obtained from clinical trial data of one clinical participant are shown in Table 1. Each value for the PPWV and PBFV is shown in Table 1. Table 1 shows $\Delta \delta=$ SRT (systolic reflection Time) $=$ S.time - R.time, $\Delta \tau=$ APT (arterial photoplethysmography time) $=$ S.time - PPG peak time, $L_{H}=$ Hand Length, $\mathrm{PPWV}=\left(L_{H} / \Delta \delta\right) \times 2, \mathrm{PBFV}=\left(L_{H} / \Delta \tau\right)$ from calculated the average values for the five continuous sections for pulse waveforms.

As shown in Table 1, the values of PBFV and PPWV measured by the simultaneous system composed of a cliptype pulsimeter and a PPG were about $1.25-1.52 \mathrm{~m} / \mathrm{s}$ and $0.27-0.50 \mathrm{~m} / \mathrm{s}$, respectively. If two parameters added to the measuring index of living body signal by instrument, it may help the diagnosis or treatment of cardiovascular patients because PBFV and PPWV display the status of a person's artery and blood flow.

The pulse diagnosis as measured in oriental medicine lacks objectivity because it is measured by the balance sense of a doctor. A prototype clip-type pulsimeter made of a Hall device and a permanent magnet that can measure changes in a magnetic field is expected to overcome this shortcoming. The voltage signal represents the kinetic energy that occurs with the motion of the pulse when attached in the "Chwan" positions and can be quantified using a Hall device situated over the radial artery. The analyses of PPWV and PBFV were possible because of the simultaneous measurement system composed of a clip-type pulsimeter and a PPG. The clip-type pulsimeter

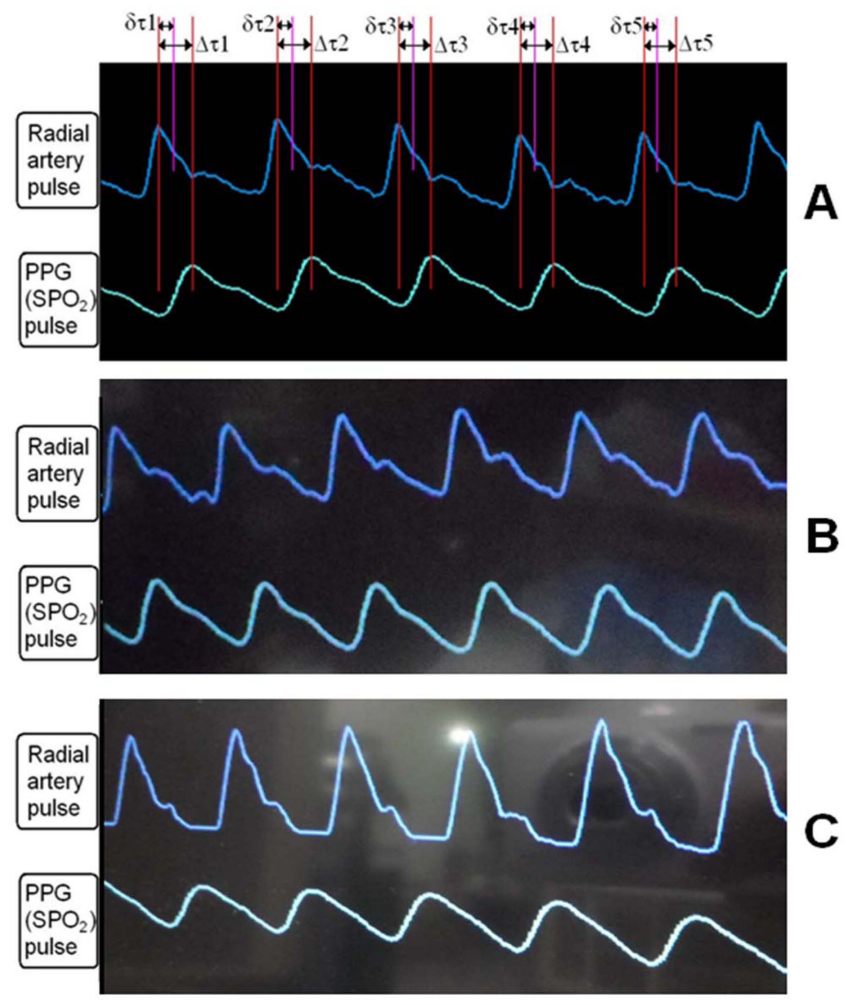

Fig. 4. (Color online) Radial artery pulse graph and PPG signals for three participants $(\mathrm{A}, \mathrm{B}$, and $\mathrm{C}$ ) obtained from the simultaneous measurements by the clip-type pulsimeter and PPG meter mounted on the wrist and fingertip of the left hand, respectively. Here, $\Delta \tau_{i}$ and $\delta \tau_{i}$ are the time interval measured from $i^{\text {th }}$ phase difference of the two pulse waveforms and the time interval between the systolic peak time and the reflective peak time in $\mathrm{i}^{\text {th }}$ radial artery pulse wave, respectively.

and PPG system is expected to improve upon the traditional oriental-western fusion medical storage medium and fusion patient monitor used in the ubiquitous health care system.

\section{Conclusion}

One prototype product of a clip-type pulsimeter equipped with a magnetic field-sensing semiconductor Hall device with one permanent magnet attached in the "Chwan" position in the center of a radial artery was developed. The clip-pulsimeter was composed of a hardware system to measure voltage signals. To measure the PBFV, the radial artery pulsimeter was simultaneously connected to the PPG. Analysis and comparison of two pulse waves' data have been obtained from a clinical test of forty subjects of 20 ages. The blood velocity simultaneously measured from an RAP wave and PPG was had an average value of $0.27-0.50 \mathrm{~m} / \mathrm{s}$. The PPWV had a 
Table 1. The measured values of systolic reflection time, arterial photoplethysmography time, hand length, peripheral pulse wave velocity (PPWV), and peripheral blood flow velocity (PBFV) obtained from clinical trial data of one clinical participant.

\begin{tabular}{|c|c|c|c|c|c|c|}
\hline $\begin{array}{l}\text { Participant } \\
\text { Name }\end{array}$ & $\begin{array}{c}\text { Wave } \\
\text { number }\end{array}$ & $\begin{array}{c}\Delta \delta^{\mathrm{a}} \\
{[\mathrm{ms}]}\end{array}$ & $\begin{array}{c}\Delta \tau^{b} \\
{[\mathrm{~ms}]}\end{array}$ & $\begin{array}{c}L_{H}^{\mathrm{c}} \\
{[\mathrm{cm}]}\end{array}$ & $\begin{array}{c}\mathrm{PPWV}^{\mathrm{d}} \\
{[\mathrm{m} / \mathrm{s}]}\end{array}$ & $\begin{array}{c}\mathrm{PBFV}^{\mathrm{e}} \\
{[\mathrm{m} / \mathrm{s}]}\end{array}$ \\
\hline \multirow{6}{*}{ A } & 1 & 230.0 & 320.0 & 15.0 & 1.30 & 0.46 \\
\hline & 2 & 248.0 & 324.0 & 15.0 & 1.20 & 0.46 \\
\hline & 3 & 243.0 & 310.0 & 15.0 & 1.23 & 0.48 \\
\hline & 4 & 244.0 & 323.0 & 15.0 & 1.23 & 0.46 \\
\hline & 5 & 234.0 & 326.0 & 15.0 & 1.28 & 0.46 \\
\hline & Average & 239.8 & 320.6 & 15.0 & 1.25 & 0.46 \\
\hline \multirow{6}{*}{ B } & 1 & 255.0 & 314.0 & 16.0 & 1.25 & 0.50 \\
\hline & 2 & 258.0 & 312.0 & 16.0 & 1.24 & 0.51 \\
\hline & 3 & 254.0 & 315.0 & 16.0 & 1.26 & 0.50 \\
\hline & 4 & 256.0 & 313.0 & 16.0 & 1.25 & 0.51 \\
\hline & 5 & 257.0 & 316.0 & 16.0 & 1.24 & 0.50 \\
\hline & Average & 256.0 & 314.0 & 16.0 & 1.25 & 0.50 \\
\hline \multirow{6}{*}{$\mathrm{C}$} & 1 & 220.0 & 610.0 & 17.0 & 1.54 & 0.27 \\
\hline & 2 & 223.0 & 612.0 & 17.0 & 1.52 & 0.27 \\
\hline & 3 & 226.0 & 620.0 & 17.0 & 1.50 & 0.27 \\
\hline & 4 & 228.0 & 613.0 & 17.0 & 1.49 & 0.27 \\
\hline & 5 & 220.0 & 615.0 & 17.0 & 1.54 & 0.27 \\
\hline & Average & 223.4 & 614.0 & 17.0 & 1.52 & 0.27 \\
\hline
\end{tabular}

${ }^{\mathrm{a}} \Delta \delta=$ systolic reflection time $=$ S.time - R.time, ${ }^{\mathrm{b}} \Delta \tau=$ artery photoplethysmography time $=$ S.time $-\mathrm{PPG}$ peak time, ${ }^{\mathrm{c}} L_{H}=$ hand length, ${ }^{\mathrm{d}} \mathrm{PPWV}$ (peripheral pulse wave velocity) $=\left(L_{H} / \Delta \delta\right) \times 2$, ${ }^{\mathrm{e}} \mathrm{PBFV}$ (peripheral blood flow velocity) $=\left(L_{H} / \Delta \tau\right)$

wider scope, with an average value of $1.25-1.52 \mathrm{~m} / \mathrm{s}$. The new method provided more precise values of PWV than the conventional biomedical signal monitoring system, implying that the data measured by this oriental medical diagnosis apparatus may be clinically useful in the future. Both PPWV and PBFV are important measurements that may help normal people understand their health status and motivate them to take better care of themselves. Predicting a blood vessel's elasticity from the analysis of PPWV may be useful for developing an oriental-western diagnostic medical signal device for the ubiquitous health care system in the future. Also, the results of this research may be used to store biomedical signals for health care system.

\section{Acknowledgments}

This study was supported by a grant from the Ph.D. Course Research Fund Program of Graduation of Sangji University and a grant of the Oriental Medicine Advanced Technology R\&D Project, Ministry for Health, Welfare \& Family Affairs, Republic of Korea (B100030).

\section{References}

[1] S. S. Lee, D. H. Nam, Y. S. Hong, W. B. Lee, I. H. Son, K. H. Kim, and J. G. Choi, Sensors 11, 1784 (2011).

[2] D. H. Nam, W. B. Lee, Y. S. Hong, and S. S. Lee, Sensors 13, 4714 (2013).

[3] R. Asmar, A. Benetos, G. M. London, C. Hughe, Y. Weiss, J. Topouchian, B. Laloux, and M. Safar, Blood Pressure 4, 48 (1995).

[4] Q. Yu, J. Zhou, and Y. C. Fung, Am. J. Physiol. Heart Circ. Physiol. 265, 52 (1993).

[5] M. F. P. O'Rourke, R. P. Kelly, and A. P. Avolio, The Arterial Pulse, 1st Ed.; Lea \& Febiger, Philadelphia, PA, USA (1992).

[6] A. Yamashina, H. Tomiyama, T. Arai, Y. Koji, M. Yambe, H. Motobe, Z. Glunizia, Y. Yamamoto, and S. Hori, Hypertens. Res. 26, 801 (2003).

[7] J. C. Bramwell and A. V. Hill, Proceedings of the Royal Society, London (1922) p. 298.

[8] John Allen, Photoplethysmography and its Application in Clinical Physiological Measurement 28, R1 (2007).

[9] K. Shelley and S. Shelley, Pulse Oximeter Waveform: Olethysmography, in Clinical Monitorning, Carol Lake, R. Hines, and C. Blitt, Eds.: W. B. Saunders Company (2001) p. 420.

[10] A. Fronek, Noninvasive diagnosis in vascular disease, McGraw Hill, New York (1989).

[11] I. H. Son and S. S. Lee, J. Korean Magn. Soc. 21, 104 (2011).

[12] M. C. Ahn, J. G. Choi, I. H. Son, S. S. Lee, and K. H. Kim, J. Korean Magn. Soc. 20, 106 (2010).

[13] S. S. Lee, M. C. Ahn, and S. H. Ahn, J. Magn. 14, 132 (2009).

[14] S. S. Lee, I. H. Son, J. G. Choi, D. H. Nam, Y. S. Hong, and W. B. Lee, J. Korean Phys. Soc. 58, 349 (2011). 